\title{
MÉTIS : RUSE, INTELLIGENCE, BRICOLAGE DANS LES SYSTÈMES COMPLEXES ET LES CRISES
}

\author{
Author(s) / Auteur(s) : \\ Alexandre MAKAROVITSCH \\ Title(s), Academic(s) position(s)... \\ University, Faculty, Society... \\ amakarovitsch@gmail.com
}

\author{
Abstract / Résumé : \\ ???. \\ Keywords / Mots-clés : \\ système, metis, ruse, complexité, bricolage, frontières, leadership
}

\section{INTRODUCTION}

Dans la trousse à outils qui devient indispensable dès qu'on approche des systèmes complexes, que ce soit pour les étudier, pour prévoir ou pour agir, les méthodes classiques deviennent insuffisantes. Les nouvelles méthodes, notamment le flou, les automates cellulaires, les simulateurs, doivent dorénavant intégrer cet ensemble, mais ils peuvent être aussi insuffisants. Une autre série d'outils : la Metis, les jeux de différents types, les moyens de recherche créative, le bricolage, viendront compléter utilement l'outillage.

Les pages qui suivent essaient de donner une vue générale de la Metis et de son utilisation dans l'approche des systèmes complexes, et notamment de ceux en crise.

Le nom de Metis est celui de la déesse grecque de la ruse, moins bien connue. Fille d'Oceanos et de Thetis, mère d'Athena (conçue avec Zeus). Elle est figurée sur les objets d'art de l'époque assise sous le trône de Zeus. La mythologie dit qu'elle est la conseillère très écoutée par Zeus.

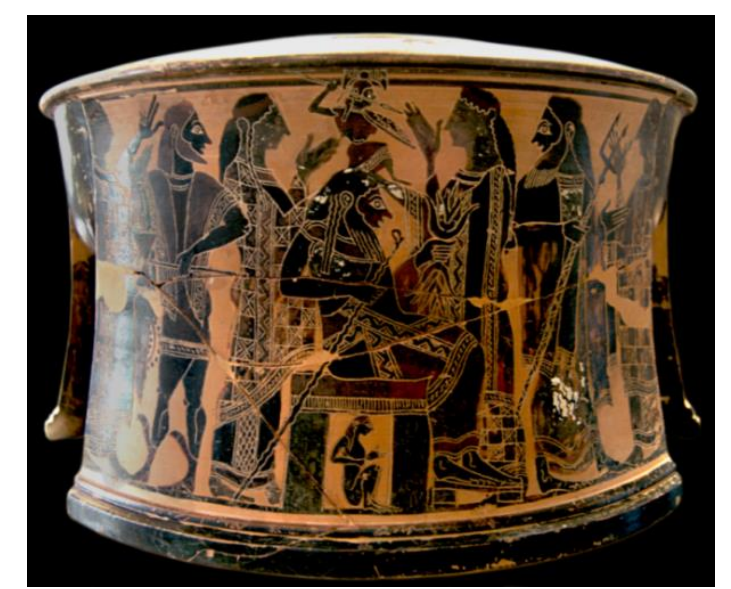

La déesse Metis est figurée accroupie sous le trône de Zeus

Pendant des siècles, la ruse, même si elle a été maintes fois utilisée, n'a pas été considérée comme un moyen convenable pour résoudre les problèmes posés par la complexité. Elle a été, dans la civilisation occidentale y compris depuis les Romains, considérée comme un défaut. En Orient, elle a été utilisée 
quasi-systématiquement dans la résolution de problèmes de toute sorte (voir par exemple «l'art de la guerre » de Sun Tzu).

Depuis peu elle est reconnue comme qualité et fait partie des outils pour approcher les systèmes complexes et résoudre certains problèmes qui s'y rapportent.

\section{LA METIS}

La Metis est un sous-système du système de la mythologie, mis en place par les grecs. Dans la mythologie, chaque dieu a une place plutôt précise avec des pouvoirs qui se superposent assez peu sans pour cela éliminer la possibilité de concurrence. Au sommet du système on trouve Zeus.

La citation ci-après (livre de Marcel Detienne et Jean-Pierre Vernant «Les ruses de l'intelligence»), nous a semblé une bonne définition du système Metis : «La Metis est bien une forme d'intelligence et de pensée, un mode du connaitre; elle implique un ensemble complexe, mais très cohérent, , d'attitudes mentales, de comportement intellectuels qui combinent le flair, la sagacité, la prévision, la souplesse d'esprit, la feinte, la débrouillardise, l'attention vigilante, le sens de l'opportunité, des habiletés diverses, une expérience longuement acquise; elle s'applique à des réalités fugaces, mouvantes, déconcertantes et ambiguës, qui ne se prêtent ni à la mesure précise, ni au calcul exact, ni au raisonnement rigoureux. » .

Il s'agit d'une multiplicité de plans mouvants sur lesquels se déplace l'esprit dans l'effort d'approcher la réalité et d'agir ou prévoir. Le plan du langage est l'un des plus évidents, avec le flou qui le caractérise. L'art du barreur dans son voilier est exprimé dans l'incertitude et l'imprécision de l'environnement marin.

D'autres mots encore, font partie de ce système de la Métis : sagesse, pouvoir, créativité, oubli, frontière, spontanéité.

La Metis met en musique tout cet ensemble, d'où sa propre complexité. Pour approcher utilement un système complexe il est nécessaire d'avoir dans l'outillage une diversité au moins égale à celle du système étudié. La Metis répond à cette nécessité en aidant à s'adapter aux situations sans les contraindre à soi.

Les qualités qui semblent nécessaires pour l'utilisation efficace de la Metis sont :

- ouverture aux idées des autres,

- pluridisciplinarité,

- forte curiosité,

- acceptation du flou, de l'imprécis, de l'incertain,

- support actif de l'expérimentation,

- acceptation de la possibilité d'absence de solution,

- créativité,

- confiance, entêtement et patience,

- habileté, souplesse et adaptabilité,

- capacité de contournement.

Il est évident que pour réunir toutes ces qualités il est impératif de travailler en équipe, et pas n'importe quelle équipe, mais une équipe solidement formée et rodée dans la tâche d'approche de la complexité. Les positions d'une telle équipe face au système complexe peuvent être: observateur et/ou acteur intervenant dans le système (étude, confrontation, exploration, accompagnement, prévision, action ponctuelle). 


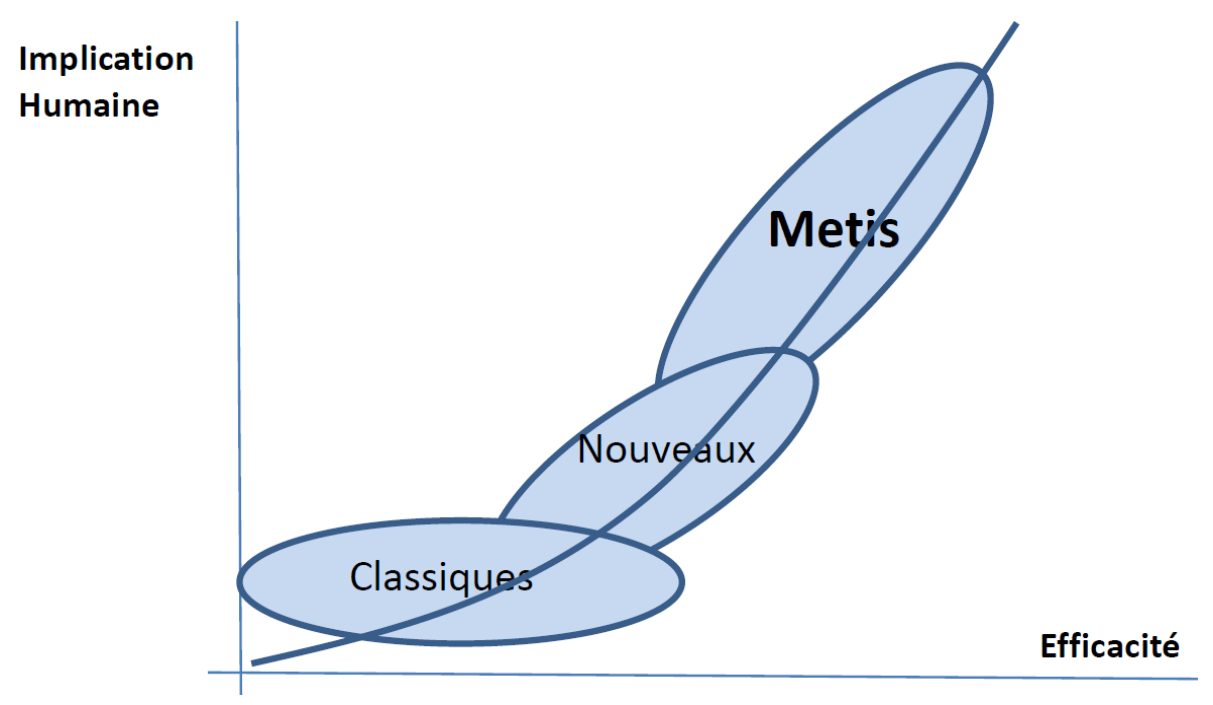

Plus généralement, pour travailler avec les systèmes complexes, trois classes d'outils prouveront leur efficacité dans des cas différents d'implication humaine, comme illustré dans le schéma ci-dessus.

Les outils classiques sont basés sur les mathématiques : statistiques, probabilités, systèmes d'équation de types divers.

Les nouveaux outils basés sur des mathématiques sont notamment : probabilités de Bayes, ensembles et logique floues, possibilités, automates cellulaires, algorithmes génétiques, simulateurs divers, systèmes à agents, visualisations, analyse synchronique/diachronique.

Enfin, les outils nouveaux différents, basés sur la Metis : analyses psychologiques, ruse, mensonge, bricolage, jeux.

La panoplie Metis implique aussi : la culture, l'expérience, diverses heuristiques, manipulation des frontières, «pifométrie », expériences de pensée.

Le mensonge et les simulacres sont des outils quelques fois, très efficaces. La revue National Geographic (numéro de juin 2017) montre une statistique fort instructive d'utilisation du mensonge : Plus de la moitié des mensonges sont utilisés pour se protéger ou pour tirer divers avantages personnels.

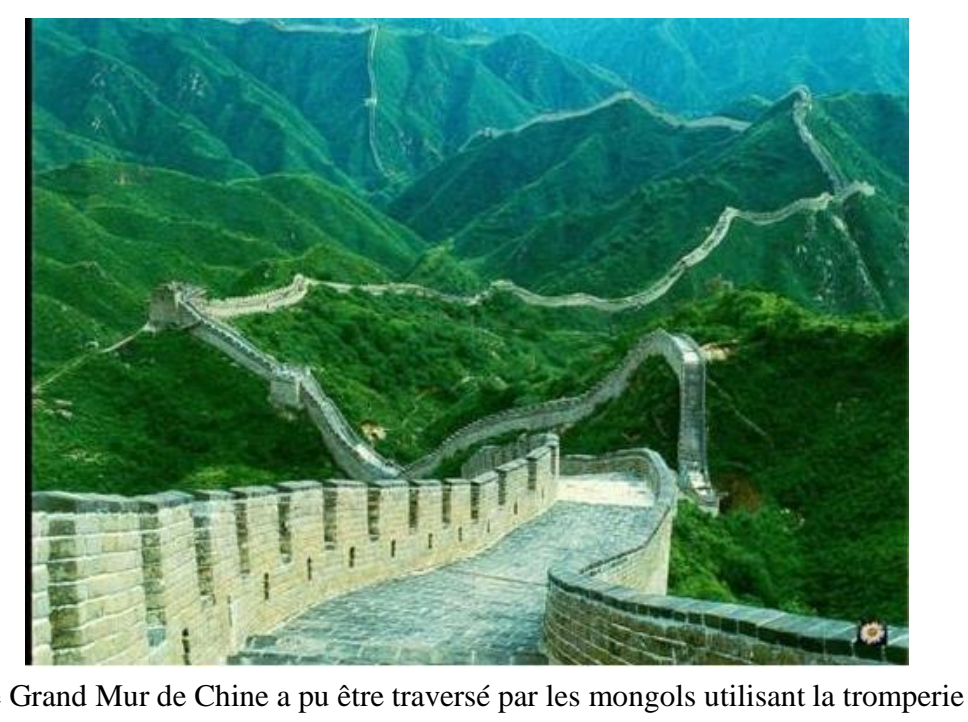

Le bricolage (qui est aussi une des attributions d'un dieu des grecs anciens, Hephaïstos), est aussi un composant de la Metis. Les caractéristiques spécifiques du bricolage sont notamment : l'essai/erreur, 
l'artisanat, l'utilisation décalée des matériaux, l'expérimentation, la recombinaison, la préparation à l'imprévu. Les caractéristiques du bricoleur sont entre autres : la passion, la curiosité, la manualité, la patience, l'habileté, la créativité, l'entêtement.

La manipulation des frontières est une façon de réduire la complexité d'un système, ou de mieux le positionner en vue de l'étude ou de l'intervention. Il s'agit construire et de déconstruire des frontières qui sont de types très différents : fixes, mobiles, floues, incertaines, matérielles, virtuelles... Les frontières sont en même temps des freins et des stimulateurs quand il s'agit de les traverser.

\section{LES CRISES}

Tous les systèmes complexes sont de façon permanente en crise. Une crise est la situation de certains composants ou interactions du système qui perturbent son fonctionnement normal. Les crises sont de durée, intensité et localisation variable. Elles peuvent avoir une solution, mais pas toujours. Elles prennent fin soit par la solution, soit de façon naturelle, soit par la destruction du système. Enfin, elles peuvent donner naissance à des émergences, non -prévisibles, positives ou négatives.

Il est intéressant d'approcher la situation de crise sous l'angle de la Metis. Ceci, quand l'implication humaine dans le système est très importante ou quand d'autres méthodes - classiques ou nouvelles s'avèrent inopérantes. Les Chinois nous enseignent que la crise présente en même temps du danger et de l'opportunité et de ce fait, ils sont à l'aise avec la Metis.

La position vis-à-vis du système peut être celle de l'observateur, de l'explorateur ou celle d'intervenant direct ou indirect. La Metis implique d'essayer l'adaptation plutôt que la confrontation/lutte, ou l'abandon/fuite.

Pour résoudre des problèmes posés par les crises des systèmes complexes il est nécessaire que l'équipe qui s'y attèle ait certaines qualités, comme : ouverture aux idées des autres, attitude favorable à la pluridisciplinarité, acceptation du flou, de l'incertitude et de l'imprécision, support actif de l'expérimentation, acceptation de la possibilité de manque de solution. Et les membres de l'équipe devraient être créatifs, travailleurs, patients, adaptables, souples, habiles et expérimentés, rigoureux.

\section{EXEMPLES}

Les quelques exemples très généraux qui suivent, montrent l'utilité de la Metis pour la résolution de problèmes complexes.

\section{Les « systèmes drones »}

Les drones peuvent être vus comme un très large système, a frontières mouvantes et grande implication humaine. Qu'ils soient civils ou militaires, ils sont de plus en plus nombreux et performants (plus autonomes, plus rapides, utilisant des technologies de plus en plus avancées). Les progrès réalisés depuis 1935 quand la société de Havilland a présenté le premier avion sans pilote ont été énormes.
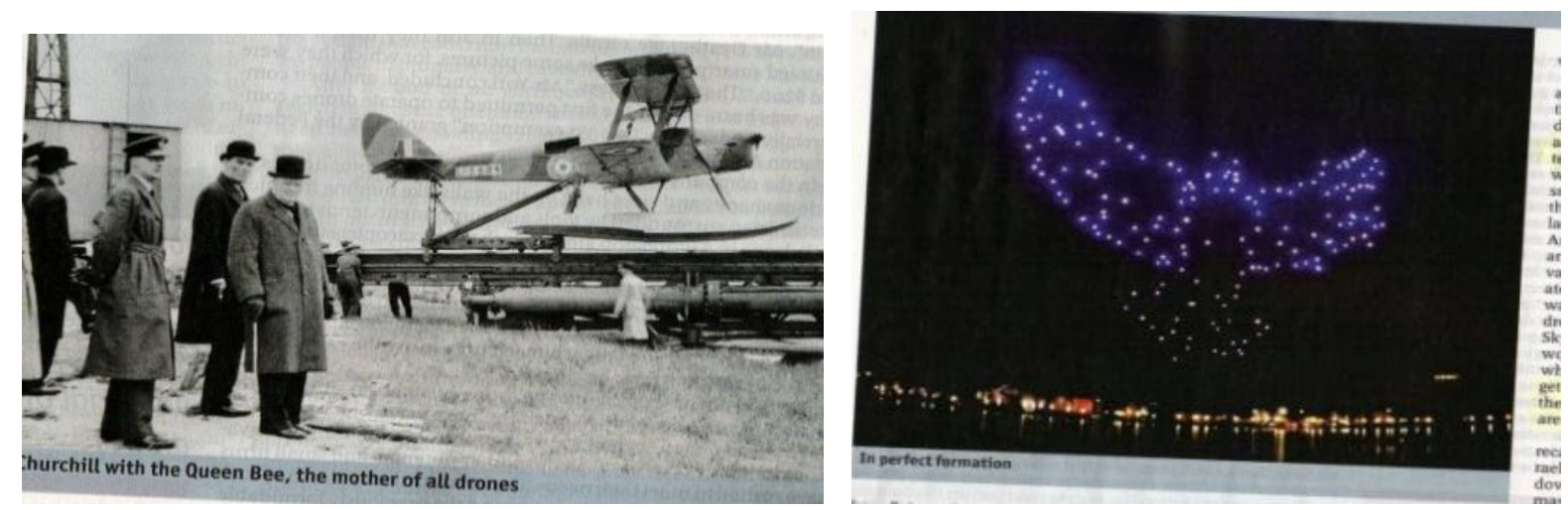
Aujourd'hui leur dimension va depuis la taille d'une mouche équipée de moyens de captage jusqu'à des drones militaires de grande taille qui peuvent porter des charges importantes et de l'armement. Ils sont aussi de plus en plus nombreux et utilisés à des fins très différentes (depuis les loisirs, en passant par la logistique, jusqu'aux attaques militaires). Ils peuvent voler en formations de plusieurs centaines.

Ils provoquent des crises quant à leur utilisation : violation de domaines privés, utilisation militaire, problèmes psychologiques, divers problèmes de sécurité ou d'imprécision et d'incertitude, voire d'absence de législation.

Approcher ces problèmes divers, nécessite souvent l'utilisation de méthodes non classiques, notamment dans le domaine légal et éthique (ou les intéressés sont loin d'avoir les mêmes objectifs), ou dans celui psychologique (le cas des pilotes qui sont déconnectés de la réalité quand ils dirigent les engins avec un joystick depuis des endroits loin du terrain et causent de gros dégâts). Ils posent aussi de nombreux problèmes liés à leur contrôle (par exemple ils peuvent être piratés et dévoyés de leur objectif). Ils sont devenus incontournables dans notre civilisation et il devient impératif d'être en mesure de bien connaitre et si possible gérer ce système.

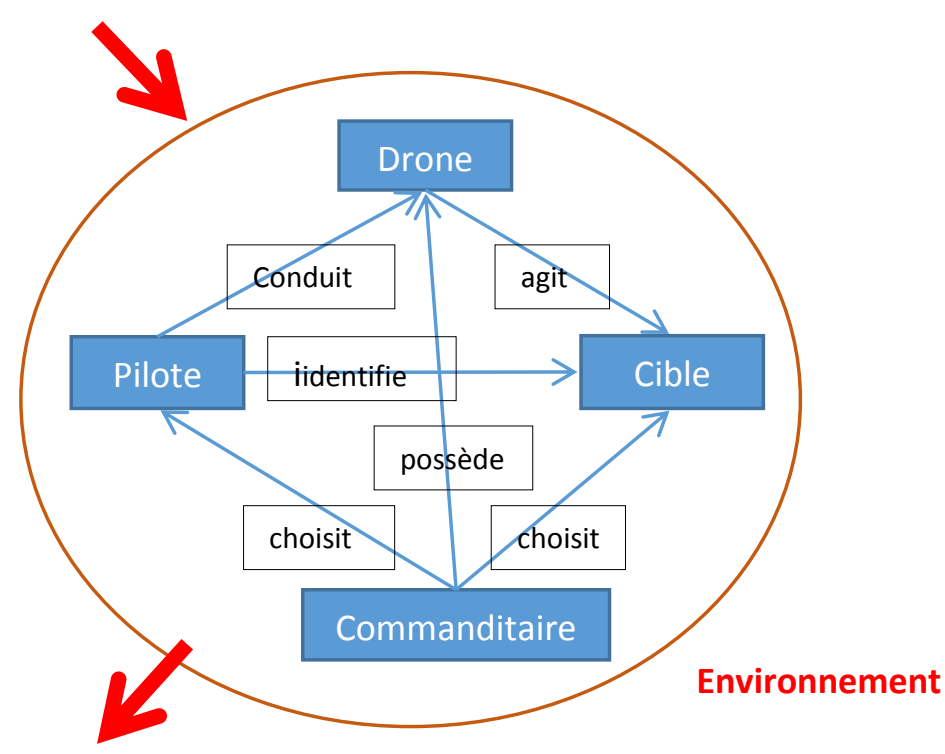

\section{LE « SYSTEME LEADERSHIP »}

Le domaine du leadership est traité ailleurs de façon quasiment exhaustive. Il mérite d'être vu aussi sous l'angle du système en crise, surtout aujourd'hui quand le leader est de plus en plus confronté à des questions difficiles notamment dans les entreprises. Doit-on se concentrer sur le court-terme ou sur le long terme ? Doit-on respecter des frontières ou les passer ? Doit-on créer de la valeur surtout pour les actionnaires ou pour les autres intéressés (salariés, partenaires, clients, « cité ») ?...

Il s'agira de prendre en considération des alternatives qui sont interdépendantes et en même temps contradictoires. Prendre en compte des vérités multiples, très différentes et souvent conflictuelles n'est pas du tout aisé, comme d'ailleurs le fait de connecter et séparer à la fois des forces opposées. Mais aussi faire face aux changements sans les combattre. L'équilibre dynamique d'un projet ou d'une entreprise est toujours un challenge. 
Un leader qui gère mieux les situations paradoxales est - comme le montre l'article de W.K.Smith \&Al dans la revue HBS de Juin-Juillet 2017 - toujours dans la position du « et ». C'est-à-dire qu'en matière de vérité il suppose que les choses sont souvent divergentes et intègrent des perspectives multiples, donc il va s'engager dans des stratégies contradictoires, va accepter des cultures différentes, va tirer des enseignements de plusieurs points de vue. Pour ce qui est du capital humain et des ressources il considèrera que ceux-ci vont pouvoir se développer et générer de nouveaux relais de croissance. Il explorera de nouvelles voies (technologies, partenariats, «coopétition »), sera souple dans la gestion des priorités et sera ouvert aux opportunités qui se présentent. Dans la pratique du leadership, il s'agira de s'adapter au changement et de l'accompagner. Cela veut dire en fait, de vivre avec l'incertitude et l'imprécision, d'expérimenter continuellement, d'agir selon plusieurs stratégies en simultané, avoir toujours des solutions provisoires prêtes à l'emploi.

Tout ceci devient de plus en plus nécessaire car l'entreprise est submergée par de données, qui constituent en même temps un risque et une opportunité. Comment manager et valoriser les données ? Comment protéger les données ? Comment exploiter au mieux les données ? Ces questions et bien d'autres ne trouveront de solutions que si le leader saura se servir au mieux d'une panoplie d'outils dont la Metis n'est pas le moins important.

\section{CONCLUSION (PROVISOIRE)}

Les systèmes complexes ayant une forte implication humaine sont en crise de façon permanente. Ces crises sont diverses du point de vue de la localisation, du déclanchement, de la durée, de l'intensité. Elles ne peuvent pas être toujours résolues, mais en tout cas il est nécessaire d'avoir à disposition de nombreuses méthodes et outils pour essayer de les résoudre : classiques ou nouveaux, dont la Metis.

Il faut savoir se déplacer confortablement dans les dimensions multiples de ces systèmes, et l'une des méthodes les plus efficaces est la Metis.

Dans ce cadre, le travail, que ce soit d'étude ou de résolution de problèmes, devient sûrement plus efficace si il est réalisé en équipe.

Les nombreux échecs dans l'utilisation uniquement des méthodes et outils classiques, devraient permettre une mutation naturelle vers l'utilisation des outils nouveaux, ainsi que de la Metis.

Ceci dit, il reste encore à mettre en place des formations adéquates qui devront commencer par la formation à l'approche et à la pensée systémiques. 